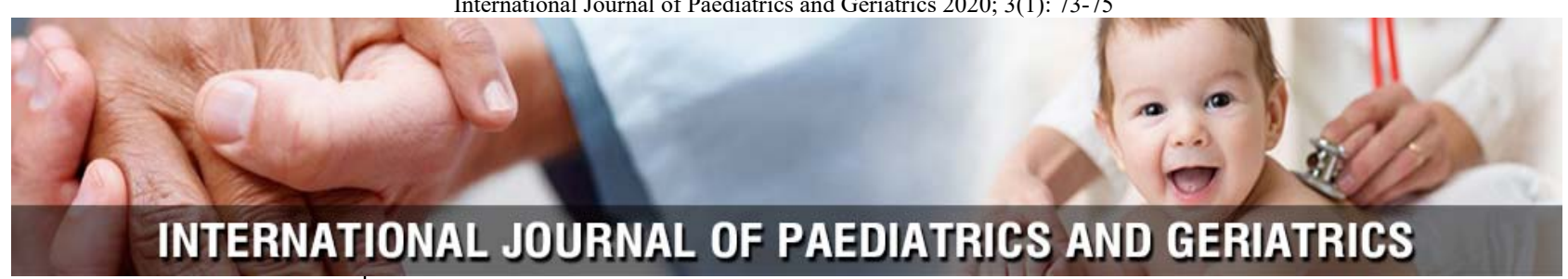

P-ISSN: 2664-3685 E-ISSN: $2664-3693$

www.paediatricjournal.com IJPG 2020; 3(1): 73-75

Received: 04-11-2019

Accepted: 08-12-2019

Dr. Radesh Pathak Associate Professor, Department of Pediatrics Varun Arjun Medical College and Rohilkhand Hospital Shahjahanpur, Uttar Pradesh, India

Dr. Sunil Kumar Agrawal Assistant Professor, Department of Pediatrics Varun Arjun Medical College and Rohilkhand Hospital Shahjahanpur, Uttar Pradesh, India

Corresponding Author: Dr. Sunil Kumar Agrawal Assistant Professor, Department of Pediatrics Varun Arjun Medical College and Rohilkhand Hospital Shahjahanpur, Uttar Pradesh, India

\section{Role of antibiotics in treatment of diarrhea in school going children}

\section{Dr. Radesh Pathak and Dr. Sunil Kumar Agrawal}

DOI: https://doi.org/10.33545/26643685.2020.v3.i1b.61

\begin{abstract}
To assess the efficacy of different antibiotic regime in treatment of diarrhoea of paediatric patients. Total of 40 paediatric patients with present of diarrhoea were included in the present study. All the patients were divided into two study groups with 20 patients in each group. Group 1 included subjects who were treated with ceftriaxone therapy while group2 included subjects who were treated with ciprofloxacin therapy. Microbial stool cultures were used as a standard for assessing the efficacy of both the antibiotic regimens. All the results were recorded in Microsoft excel sheet and were analyzed by SPSS software.

Results: Mean age of the patients of group 1 and group 2 was 15.8 years and 14.9 years respectively. There were 12 males and 8 females in group 1 while there were 11 males and 9 females in group 2 . Success rate among both the study groups was found to be 95 percent. Non- significant results were obtained while comparing the success rate of both the antibiotic treatment therapies.

Conclusion: Both ceftriaxone and ciprofloxacin therapy are equally effective in treating diarrhoea in paediatric patients.
\end{abstract}

Keywords: Regime, antibiotic, diarrhoea, paediatric patients, ceftriaxone, ciprofloxacin

\section{Introduction}

One of the major health problem encountered worldwide among children is Diarrheal disorders. Accounting for more than 750,000 deaths in children under the age of 5 per year, they are the second leading cause of death in this population according to the World Health Organization (WHO) ${ }^{[1,2]}$.

Fluid and electrolyte therapy is the mainstay of therapy. Antiemetic, antibiotic and antidiarrheal medications can usually be avoided. Commercial clear fluid products have been reformulated to allow effective rehydration and maintenance of hydration, thus avoiding hospitalization or use of intravenous therapy. Patients are quickly advanced to nutrient feedings using breast milk or formula ${ }^{[3-5]}$. Hence; we planned the present study to assess the efficacy of different antibiotic regime in treatment of diarrhoea of paediatric patients.

\section{Subjects and Methods}

The present study was conducted in the department of paediatric medicine of the medical institute and it included assessment of efficacy of different antibiotic regime in treatment of diarrhoea of paediatric patients. A total of 40 paediatric patients with present of diarrhoea were included in the present study. All the patients were divided into two study groups with 20 patients in each group. Group 1 included subjects who were treated with ceftriaxone therapy while group2 included subjects who were treated with ciprofloxacin therapy. Microbial stool cultures were used as a standard for assessing the efficacy of both the antibiotic regimens. All the results were recorded in Microsoft excel sheet and were analysed by SPSS software. Chi- square test was used for assessment of level of significance. P- value of less than 0.05 was taken as significant.

\section{Results}

In the present study, analysis of a total of 40 patients was carried out. Among these 40 patients, 20 were given ceftriaxone therapy while the remaining 20 were given ciprofloxacin therapy. Mean age of the patients of group 1 and group 2 was 15.8 years and 14.9 years respectively. There were 12 males and 8 females in group 1 while there were 11 males and 9 females in group 2. 
Table 1: Demographic Data

\begin{tabular}{|c|c|c|}
\hline Parameter & Group 1 & Group 2 \\
\hline Mean age (years) & 15.8 & 14.9 \\
\hline Male & 12 & 11 \\
\hline Female & 8 & 9 \\
\hline
\end{tabular}

Table 2: Positive stool cultures before and after treatment

\begin{tabular}{|c|c|c|c|c|}
\hline & $\begin{array}{c}\text { Pre- } \\
\text { treatment }\end{array}$ & & $\begin{array}{c}\text { Post- } \\
\text { treatment }\end{array}$ & \\
\hline Positive cultures & Group 1 & Group 2 & Group 1 & Group 2 \\
\hline Salmonella & 7 & 6 & 1 & 0 \\
\hline $\begin{array}{c}\text { Vibri } \\
\text { Parahaemilyticus }\end{array}$ & 5 & 5 & 0 & 1 \\
\hline Campy lobacter & 4 & 5 & 0 & 0 \\
\hline Shigella & 2 & 3 & 0 & 0 \\
\hline Others & 2 & 1 & 0 & 0 \\
\hline Total & 20 & 20 & 1 & 1 \\
\hline
\end{tabular}

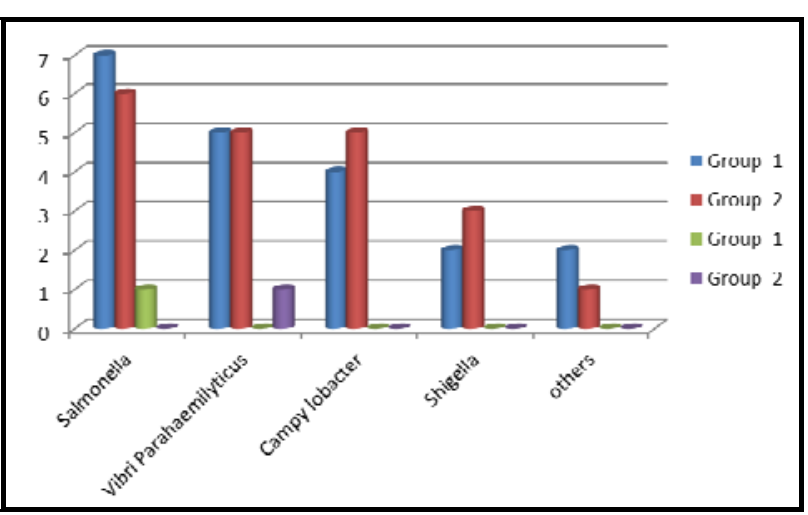

Fig 1: Positive stool cultures before and after treatment

Table 3: Comparison of success rate of both the treatment regimes

\begin{tabular}{|c|c|c|c|}
\hline Microbiological result & Group 1 & Group 2 & p-value \\
\hline Success & 19 & 19 & \multirow{2}{*}{1} \\
\hline Failure & 1 & 1 & \\
\hline
\end{tabular}

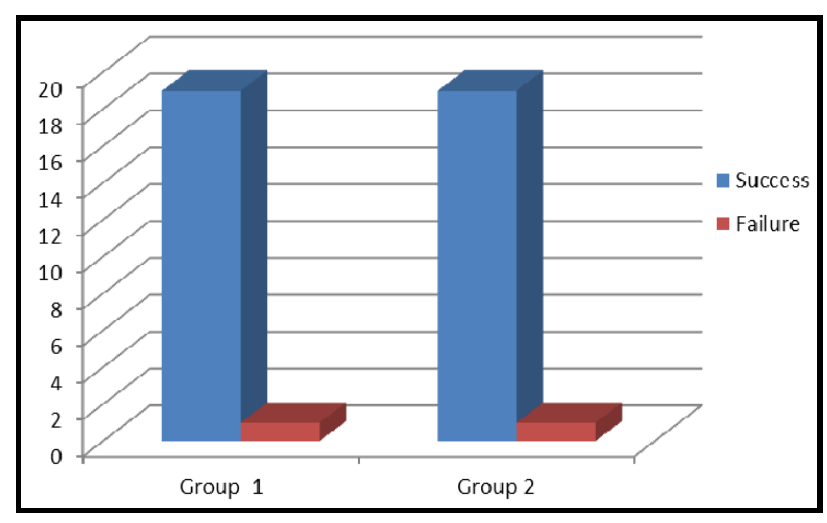

Fig 2: Comparison of success rate of both the treatment regimes

\section{Discussion}

Acute infectious enteritis (including gastroenteritis) remains one of the commonest causes of death among infants and children in developing countries. Diarrheal diseases have been classified into four clinical types: Acute watery diarrhea, acute bloody diarrhea (dysentery), persistent diarrhea, and diarrhea with severe malnutrition. Acute watery diarrhea is of sudden onset and lasts for several hours or days. The main concern is dehydration. Weight loss can occur if feeding is being withheld. Acute bloody diarrhea is considered when blood and mucous is present in the stool. Persistent diarrhea lasts 14 days or longer [6-9]. Hence; we planned the present study to assess the efficacy of different antibiotic regime in treatment of diarrhea of paediatric patients.

In present study analysis of total of 40 patients was carried out. Among these 40 patients, 20 were given ceftriaxone therapy while remaining 20 were given ciprofloxacin therapy. The etiological pattern of bacteria causing acute diarrhea depends on geographical area. In developing countries more than half a million infants and children die each year of Age and Vibrio cholera still causes epidemics, but most common bacterial agents is Shigella ${ }^{[4]}$. In the Europe, most common bacterial pathogens are Campylobacter, Salmonella, spp., Enteropathogenic E. Coli (EPEC) and Enteroaggregative E. Coli (EAEC) ${ }^{[5,6]}$. Clostridium difficile (Cd) has emerged as a cause of community-acquired diarrheal illness, but local data report a relatively a burden ${ }^{[10-12]}$.

In the present study, mean age of patients of group 1 and group 2 was 15.8 years and 14.9 years Respectively there was 12 males and 8 females in group 2. The WHO recommends treating all episodes of blood in the stools with antibiotics and to use ciprofloxacin as the first -line drug. Altemative are pivmecillinam, azithromycin, and ceftriaxone 50. This recommendation has been confirmed although in recent years the rates of resistance are increasing Fluoroquinolones are often empirically used in adults, and cephalosporins used to treat children with suspected bacterial AGE. Fluoroquinolones are effective Against a wide variety of enteric infections in adults, including shigellosis, salmonellosis, Typhoid fever, cholera, and campylobacter infections. Like all quinolones ciprofloxacin causes arthropathic effects in immature animals and their use has been limited in children ${ }^{[13,14]}$.

Success rate among both the study groups was found to be $95 \%$. Non-significant results were obtained while comparing the success rate of both the antibiotic therapies. Stool culture has been perceived as being a diagnostic tool that causes expense without often yielding useful results. In one large hospital, only $2.4 \%$ of stool cultures identified a pathogen, and in $<5 \%$ of the cases did the culture results lead to a change in therapy. Among out patients, positive cultre results have been reported in $6 \%-15 \%$ of culture. Nevertheless, diagnostic stool cultures can be important to both clinical and public health practice. Certain diarrheal illnesses respond well to properly select antimicrobial treatment [12,14]. Rogawski ET et al assessed the effect of Caregiver - reported antibiotic treatment for diarrhea on the timing of a child's next episode among 434 children followed from birth to 3 years of age in Vellore, India. We estimated median time differences and time ratios from inverse probability of exposure- weighted Kaplan - Meier curves for the time to next diarrhea episode. Comparing children who did and did not receive antibiotic for the previous episode. Study children had more than five diarrhea episodes on average in the first 3 years of life, and more than a quarter of all episodes were treated with antibiotics. Children who received antibiotic for their first diarrhea episode had their second episode on average 8 weeks earlier (Median time difference: $-8,95 \%$ confidence interval: $-10-3$ ) than children who did not receive antibiotics. The effects of antibiotics on subsequent diarrhea 
were greatest at earlier episode and younger ages and cefixime had a slightly larger effect compared with cotrimoxazole. Antibiotic treatment of diarrhea was associated with reduced time to a subsequent diarrhea episode especially among younger infants.

\section{Conclusion}

Under the light of above obtained results, the authors conclude that both ceftriaxone and ciprofloxacin therapy are equally effective in treating the diarrhea in paediatric patients. However further studies are recommended.

\section{Source of support: Nil}

Conflict of interest: Not declared

\section{References}

1. World Health Organization: Diarrheal disease. Reference Source, 2013.

2. Johnston BC, Shamseer L, da Costa BR et al. Measurement issues in trials of pediatric acute diarrheal diseases: A systematic review. Paeditrics. 2010; 126(1):e222-31.

3. Listernick R, Zieserl E, Davis AT. Outpatient oral rehydration in the United States, Am J Dis Child. 1986; 140(3):211-5.

4. Guarino A1, Albano F, Ashkenazi S et al. European Society for Paediatric Gastroenterology, Hepatology, and Nutrition/European Society for Paediatric Infectious Diseases evidence-based guidelines for the management of acute gastroenteritis in children in Europe. J Pediatr Gastroenterol Nutr. 2008; 46(2):S81122.

5. Van Eijk AM, Brooks JT, Adcock PM et al. Diarrhea in children less than two years of age with known HIV status in Kisumu, Kenya. Int J Infect Dis. 2010; 14(3):e220-5.

6. Buccigrossi V, Laudiero G, Nicastro E et al. The HIV-1 transactivator factor (Tat) induces enterocyte apoptosis through a redox-mediated mechanism. PLoS One. 2011; 6(12):e29436.

7. Trehan I, Goldbach HS, LaGrone LN, et al. Antibiotics as part of the management of severe acute malnutrition. Malawi Med J. 2016; 28(3):123-130.

8. Tamer AM, Friedman LB, Maxwell SR, Cynamon HA, Perez HN, Cleveland WW. Oral rehydration of infants in a large urban U.S. medical center. J Pediatr. 1985; 107(1):14-9.

9. Soriano-Gabarró M, Mrukowicz J, Vesikari T, Verstraeten T. Burden of rotavirus disease in European Union countries. Pediatr Infect Dis J. 2006; 25(1):S7S11.

10. Guidelines for the control of shigellosis, including epidemic due to Shigella dysenteriae type 1. Geneva, reference source, 2005.

11. Lo Vecchio A, Liguoro I, Bruzzese D et al, Adherence to guidelines for management of children hospitalized for acute diarrhea. Pediatr Infect Dis J. 2014; 33(11):1103-8

12. DuPont HL. Acute infectious diarrhea in immunocompetent adults. N Engl J Med. 2014; 370(16):1532-40

13. Rogawski, Elizabeth $\mathrm{T}$ et al. Antibiotic treatment of diarrhoea is associated with decreased time to the next diarrhoea episode among young children in Vellore,
India. International journal of epidemiology. 2015; 44(3):978-87. 